\title{
Non-uniform Continuum Model for Solvated Species Based on Frozen- Density Embedding Theory: The Study Case of Solvatochromism of Coumarin 153
}

\author{
Sapana V. Shedge, Xiuwen Zhou, and Tomasz A. Wesolowski*
}

\begin{abstract}
Recent application of the Frozen-Density Embedding Theory based continuum model of the solvent, which is used for calculating solvatochromic shifts in the UV/Vis range, are reviewed. In this model, the solvent is represented as a non-uniform continuum taking into account both the statistical nature of the solvent and specific solute-solvent interactions. It offers, therefore, a computationally attractive alternative to methods in which the solvent is described at atomistic level. The evaluation of the solvatochromic shift involves only two calculations of excitation energy instead of at least hundreds needed to account for inhomogeneous broadening. The present review provides a detailed graphical analysis of the key quantities of this model: the average charge density of the solvent $\left(\left\langle\rho_{\mathrm{B}}\right\rangle\right)$ and the corresponding Frozen-Density Embedding Theory derived embedding potential for coumarin 153.
\end{abstract}

Keywords: Continuum models · Density functional theory $\cdot$ Multi-level simulations ·

Orbital-free embedding theory $\cdot$ Solvatochromism

\section{Introduction}

Reliable simulations of electronic spectra of solvated chromophores in the $\mathrm{UV} / \mathrm{Vis}$ range remain a challenging task for computational chemistry despite continuous improvements in methods, their numerical implementation, and the used hardware. Three factors combine to make the task extremely difficult: i) the large number of atoms in any realistic models of the solvated chromophore, ii) the statistical nature of the solvent which makes a simple picture of the system as a molecular cluster in a given geometry inadequate, and iii) the tough accuracy requirements for the calculated excitation energies - the error of $1 \mathrm{kcal} / \mathrm{mol}$ in energy (commonly accepted target accuracy for thermochemistry) corresponds to the change of the color of light from green to yellow. To the computational chemist's relief, the vibronic structure is usually hardly visible for organic chromophores in polar solvents and the FranckCondon approximation seems adequate. The challenge is even greater if the simu-

\footnotetext{
${ }^{\star}$ Correspondence: Prof. T. A. Wesolowski Department of Physical Chemistry University of Geneva

30, quai Ernest-Ansermet

$\mathrm{CH}-1211$ Genève 4

Tel.: +41223796101

E-Mail: tomasz.wesolowski@unige.ch
}

lation aims at modeling the shape of emission bands. Here, the quantum mechanical method used to determine the excitation energy must be able to yield adequate geometries of the solvated chromophore in the excited electronic state. Usually, it is more challenging to model excited state properties of molecular systems than their ground-state counterparts. The embedding strategy, i.e. when the quantum mechanical descriptors are used only for the chromophore (or the chromophore with a few tightly bound solvent molecules), provides a pragmatic solution at least as far as the difficulty related to the size is concerned. Embedding methods include socalled QM/MM strategies, which proved to be very useful as evidenced by the 2013 Nobel Prize in Chemistry which went to Professors Karplus, Levitt, and Warshel who were instrumental in their development, as well as continuum models. The difficulty caused by fluctuations of the solvent (and solute) geometries, i.e. the principal factor behind observed broadening of the bands, however, persists. Modeling inhomogeneously broadened bands involves at least hundreds of repetitive calculations of excitation energies for instantaneous geometries of the solute-solvent system..$^{[1-3]}$ A simplified strategy is frequently applied if the objective is only the solvatochromic shift leaving aside modeling the broadening of the bands. It can be called 'static picture of the solvated chromophore' because it relies on using one representative structure of the system. This applies to both atomistic (QM/MM for instance) or continuum models (PCM, ${ }^{[4]}$ COSMO, ${ }^{[5]}$ or the model considered in the present work). In a static picture, a 'spike spectrum' is obtained, i.e. the bands are represented as spikes with the height proportional to the calculated oscillator strengths. For the sake of graphical representation, these 'spike spectra' are frequently presented after broadening using an envelope (typically Gaussian) of the empirically adjusted width. The static picture is very attractive because only two calculations of the excitation energy of the embedded chromophore are needed in order to evaluate the solvatochromic shift. Of course, the static picture involves choosing the particular geometry of the system for the quantum chemistry calculations. The choice of this geometry is not always trivial - even if the chromophore is structurally rigid, a small average lengthening or shortening of chemical bonds occur due to the interactions with the solvent. In uniform continuum models of the solvent such as PCM, the solvent is structureless because it is represented by means of a continuum with a given (constant) dielectric. Specific solute-solvent interactions are not taken into account. The advantage of the uniform continuum model over the atomistic one lies, however, in the statistical treatment 
of the solvent. The adequacy of this model increases with decreasing role of specific solute-solvent interactions. The FrozenDensity Embedding Theory (FDET) can be applied within the atomistic strategy, ${ }^{[2,3]}$ where it can be seen as an alternative to $\mathrm{QM} / \mathrm{MM}$ simulations, or within a continuum picture of the solvent. ${ }^{[6,7]}$ This work concerns the second type of application of FDET leading to the nonuniform continuum model of the solvent. This model can be seen as an intermediate level of description lying between uniform continuum and atomistic simulations. The specific solvent-solute interactions are represented in a statistical manner. In the subsequent sections, we make a compact overview of Frozen Density Embedding Theory, present the key features of the nonuniform continuum representation of the solvent in FDET, and discuss the results in the case of solvated coumarin 153 .

\section{Frozen-Density Embedding Theory (FDET)}

Frozen-Density Embedding Theory (FDET) ${ }^{[8,9]}$ is a general formal framework for multi-level numerical simulations in which quantum mechanical descriptors such as the wavefunction are used for part of a large system. The remaining part (usually large) is described using only some non-negative function $\left(\rho_{B}\right)$, which will be referred as 'frozen density'. FDET provides self-consistent expressions for the energy and the embedded wavefunction. Here, the key features of FDET are provided. For the complete set of equations and definitions in FDET, see ref. [9]. The energy obtained in FDET $\left(\mathrm{E}_{\text {emb }}\left[\rho_{\mathrm{B}}\right]\right)$ is an upper bound for the ground-state energy of the total system $\left(\mathrm{E}_{\mathrm{o}}\right)$.

$$
\begin{aligned}
E_{e m b}\left[\rho_{B}\right] & =\min _{\rho \geq \rho_{B}} E^{H K}[\rho] \\
& =E^{H K}\left[\rho_{A}^{\min }+\rho_{B}\right] \geq E_{0}
\end{aligned}
$$

where $E^{\mathrm{HK}}[\rho]$ is the Hohenberg-Kohn density functional for the ground-state energy. ${ }^{[10]}$ By definition, $\rho$ is non-negative and the integral of $\rho$ is an integer $\left(\mathrm{N}_{\mathrm{AB}}\right)$.

In FDET, the optimal density $\left(\rho_{A}{ }^{\text {min }}\right)$ is obtained from the Euler-Lagrange Equations. If $\rho_{A}$ is represented by means of an embedded wavefunction, $\left(\rho_{A}(\vec{r})=\left\langle\Psi_{A}^{e m b}|\hat{\rho}| \Psi_{A}^{e m b}\right\rangle\right), \Psi_{A}^{e m b}$ satisfies the following equation:

$$
\left(\hat{H}_{o}+\hat{V}_{e m b}\right) \Psi_{A}^{e m b}=\varepsilon \Psi_{A}^{e m b}
$$

FDET provides system-independent expressions for the potential $\hat{V}_{e m b}$ as a functional of charge densities $\left(\rho_{A}, \rho_{B}\right.$, and charge density of the nuclei in the environment) for the Hamiltonian $\hat{H}_{o}$ either including the electron-electron repulsion or not as for a Kohn-Sham system. ${ }^{[11]}$ The FDET embedding potential reads:

$$
\begin{aligned}
& V_{e m b}^{F D E T}\left[\rho_{A}, \rho_{B} ; \vec{r}\right]= \\
& =-\sum_{\alpha}^{N_{B}} \frac{Z_{\alpha}^{B}\left|\vec{R}_{\alpha}^{B}-\vec{r}\right|}{\int}+\int \frac{\rho_{B}\left(\vec{r}^{\prime}\right)}{\left|\vec{r}-\vec{r}^{\prime}\right|} d r^{\prime}+ \\
& \left.\frac{\delta E_{x c}^{\text {nad }}\left[\rho, \rho_{B}\right]}{\delta \rho}+\left.\frac{\delta T_{s}^{\text {nad }}\left[\rho, \rho_{B}\right]}{\delta \rho}\right|_{\rho=\rho_{A}}+3\right)
\end{aligned}
$$

where $N_{B}$ is the number of nuclei in subsystem $B$ and $Z_{\alpha}{ }^{B}$ is the nuclear charge of nucleus $\alpha$ in subsystem $B$. The non-additive functionals $\mathrm{E}_{x c}{ }^{n a d}\left[\rho_{\mathrm{A}}, \rho_{\mathrm{B}}\right]$ and $\mathrm{T}_{s}{ }^{\text {aad }}\left[\rho_{\mathrm{A}}, \rho_{\mathrm{B}}\right]$ are defined as the differences $\mathrm{X}^{s}$ ad $\left[\rho_{\mathrm{A}}, \rho_{\mathrm{B}}\right]$ $=\mathrm{X}\left[\rho_{\mathrm{A}}+\rho_{\mathrm{B}}\right]-X\left[\rho_{\mathrm{A}}\right]-\mathrm{X}\left[\rho_{\mathrm{B}}\right]$, where $\mathrm{X}[\rho]$ stands for one of the density functional defined in the Levy constrained search: ${ }^{[12]}$ either exchange-correlation energy $\left(E_{x c}[\rho]\right)$ or kinetic energy in a reference system of non-interacting electrons $\left(T_{s}[\rho]\right)$.

\section{Excited States}

FDET offers two formal ways to obtain excited states: one in which the excited state solution of Eqn. (2) is used to obtain excitation energy (and density) ${ }^{[13,14]}$ or the other one ${ }^{[15]}$ based on the response theory in time-dependent density functional theory (LR-TDDFT). Concerning the first strategy, Carter and collaborators pioneered such methods based on an ad hoc combination of the FDET embedding potential with traditional methods of wavefunction-based quantum chemistry.[16] The formal justification of such combination was given only recently together with the identification of made assumptions and approximations. $[9,13,14]$ The present review concerns the LR-TDDFT strategy within FDET. We notice that each term for the embedding potential given in Eqn. (3) is either $\rho_{A}$ independent or is a functional of $\rho_{A}$. Evaluation of the functional derivative of this potential with respect to $\rho_{A}$ is, therefore, possible. As a result, the mathematical framework of LR-TDDFT ${ }^{[17]}$ is applicable also if the DFT effective potential for isolated species is modified by addition of the FDET embedding potential. In practice, it involves solving Casida equations with a modified response kernel, which includes the contribution from the embedding potential given in Eqn. (3) (see ref. [15] for details). Such straightforward extension of the ground-state FDET involves an additional approximation in LR-TDDFT - neglecting the dynamic response of the density of the environment (NDRE), i.e. imposing $\delta \rho_{\mathrm{B}}(\omega)=0$ for the frequencies $\omega$ in the investigated spectral range. The NDRE approximation is applicable only for such excitations, which are localized in the embedded subsystem (subsystem A) and the energy of which is well separated on the energy scale from the excitations in the environment. A more general formalism going beyond NDRE in LR-TDDFT was introduced by Casida and Wesolowski.[18] For computational methods based on this generalization, see the works by Neugebauer (see the recent review ${ }^{[19]}$ ). (Note that in these works FDET calculations using NDRE in LR-TDDFT are called 'uncoupled FDE').

\section{Non-uniform Continuum Model Based on FDET}

FDET equations for potential and the total energy (Eqns. (18), (24), (27), and (44) in ref. [8]) depend parametrically on the electron density associated with the environment ( $\rho_{B}$ in Eqns. (1) and (3)). Usually, this $\rho_{B}$ is generated using some less expensive quantum mechanics-based method as a ground-state electron density of some polyatomic system within the Born-Oppenheimer approximations. FDET equations hold, however, for a more general class of functions. The nonuniform continuum model considered in the present work uses the average electron density of the environment to this end. This quantity will be denoted with $\left\langle\rho_{B}>\right.$ here. $\left.<\rho_{\mathrm{B}}\right\rangle$ cannot be associated with any molecular structure. In each point of space, it represents the density of probability of finding an electron. $\left\langle\rho_{B}\right\rangle$ is not uniform and can be used as $\rho_{B}$ in all FDET equations. It reflects the enhanced or reduced probabilities of finding the solvent molecules in various areas around the solute due to specific interactions. Owing to fluctuations of the nuclear positions, $\left\langle\rho_{\mathrm{B}}\right\rangle$ is a much smoother function than a typical electron density obtained within BornOppenheimer approximation for a given instantaneous geometry of the solvent molecules. The FDET based non-uniform model of the solvent is closely related to the average electrostatic potential model of the solvent (see ref. [20] for recent applications). The latter also uses the average potential generated by the environment. Compared to FDET, the non-electrostatic components of the FDET potential are neglected. Since the electrostatic potential is linearly related to the density, averaging the potential and averaging the density is equivalent with such truncated embedding potential. This does not hold for FDET embedding potential which comprises nonlinear terms.

In ref. [6], where the FDET-based nonuniform continuum model of the solvent for solvatochromism was introduced, it 
was demonstrated that the use of $\left\langle\rho_{B}\right\rangle$ in FDET is a very efficient alternative to explicit band simulations in which the excitation energies are evaluated a few hundred of times with different $\rho_{B}$ at different geometries. For two investigated cases of solvatochromism in absorption, the $n \rightarrow \pi^{*}$ band in hydrated acetone and $\pi \rightarrow \pi^{*}$ band in hydrated aminocoumarin C151, the solvatochromic shifts obtained using the two approaches within FDET: atomistic (reported originally in ref. [2]) and non-uniform continuum (reported in ref. [6]) are practically identical. The error due to replacing hundreds of evaluations of excitation energy in the statistical approach by a single evaluation using the average density of the solvent amounts to less than $0.01 \mathrm{eV}$ which is about two orders of magnitude smaller than the magnitude of the shifts. Moreover, the calculated shifts and the experimental shifts reported in the literature in all considered solvents (water, methanol, diethyl ether, and n-hexane) and chromophores (acetone, aminocoumarin 151, acrolein, and benzophenone) were in excellent agreement (within $0.03 \mathrm{eV}$ ) except for a few cases: the $n \rightarrow \pi^{*}$ transition of aminocoumarin in methanol $(-0.21$ $\mathrm{eV}$ vs. $-0.31 \mathrm{eV}(\exp ))$, the $\mathrm{n} \rightarrow \pi^{*}$ transition for benzophenone in water $(0.16 \mathrm{eV}$ vs. $0.27 \mathrm{eV}(\exp ))$, the $\pi \rightarrow \pi^{*}$ transition for benzophenone in water $(-0.10 \mathrm{eV}$ vs. $-0.20 \mathrm{eV}(\exp ))$, and the $n \rightarrow \pi *$ transition for acrolein in water $(0.33 \mathrm{eV}$ vs. $0.25 \mathrm{eV})$. The origin(s) of these differences are currently subjects of our investigations. Zhou et al. performed a comprehensive study of the performance of this model for the $(\pi \rightarrow$ $\left.\pi^{*}\right)$ absorption band of coumarin 153 in nine solvents of different polarity. ${ }^{[7]}$ The simulations confirmed the good performance and they found very small deviations between calculated and experimental solvatochromic shifts (the average deviation equals to about $0.02 \mathrm{eV}$ ). Recently, the FDET-based non-uniform continuum model was adopted for simulation of solvatochromic shifts in emission. [21]

\section{Average Charge Densities of the Solvent and Embedding Potentials for Solvated Coumarin 153}

In this section, the average charge density and the corresponding embedding potential for both the ground- and excited state of solvated coumarin 153 in different solvents are discussed in addition to the results available in the original publications on this chromophore. ${ }^{[7,21]}$ The analyses are made for the lowest transition, which has a $\pi \rightarrow \pi^{*}$ character (the corresponding orbitals are shown in Fig. 2). In each discussed example, the average charge density $\left\langle\rho_{B}\right\rangle$ is obtained using a two-step procedure. In the first step, the classical statistical theory of liquids (3D-RISM ${ }^{[22]}$ ) is used to obtain the average occupancy of a given volume element by a given atom. In the second step, $\left\langle\rho_{B}\right\rangle$ is obtained by a convolution of these occupancies with spherically symmetric electron densities associated with each atom. Details of the calculations can be found in the original publications. ${ }^{[6,7,21]}$ All the presented plots were made using ADFview software. ${ }^{[23]}$ Fig. 1 shows the embedding potentials for coumarin 153, evaluated for the ground-state electron density and geometry of the chromophore in cyclohexane and in water. The embedding potential in both cases features a cavity corresponding to the shape of the chromophore. The envelope formed by the yellow lines reflects the shape of the molecule and reflects the intermolecular Pauli repulsion (or quantum confinement effect), which is similar for both solvents. It is slightly larger for cyclohexane than for water reflecting the larger size of the solvent molecule. The yellow edge is brighter for water than for cyclohexane indicating steeper increase of the potential due to stronger solute-solvent interactions. Within the cavity, the embedding potential is, however, qualitatively different in the two cases. For cyclohexane, the embedding potential remains almost constant in the cavity, where the embedded orbitals of the chromophore are localized (see Fig. 1c). The solvent can be expected, therefore, to affect each embedded orbital in the same manner. Indeed, the energy of the highest occupied embedded orbitals and the lowest unoccupied embedded orbitals are uniformly shifted up by about $0.01 \mathrm{eV}$. As a result, the gap is not affected by the solvent. We interpret the small upshifting of the orbital levels in this non-polar solvent as the result of the Pauli repulsion, which penalizes the overlap between the electron density of the chromophore and that of the solvent. The situation is different for water. The embedding potential varies noticeably inside the cavity. It even changes the sign in different parts of the molecule, which might lead to selective stabilization or destabilization of a particular orbital. Indeed, the energy of the highest occupied embedded orbital is lowered by about $0.4 \mathrm{eV}$ whilst that of the lowest unoccupied embedded orbital is lowered by about $0.7 \mathrm{eV}$ in water. As a result, the gap decreases, which is reflected in the negative shift (see Table 1). In principle, the embedding potential affects both the shape of the embedded orbitals and their energy. The energy differences correlate well with the solvatochromic shifts whereas the orbital shapes appear not noticeably affected by the solvent (Fig. 1). This suggests that the behavior of the embedding potential in the molecular interior should be considered in qualitative analyses of the solvatochromism.

Modeling emission spectra of solvated chromophores faces additional difficulty due to the need to take into account the effect of the electronic excitation on the geometry for both the chromophore and the solvent. In the case of the model discussed here, the structure of the solvent is taken into account in a statistical manner. Concerning the chromophore, two new effects must be considered in the context of the used FDET based non-uniform continuum model: the effect of excitation on the geometry of the solute and on the charge distribution within the chromophore (to take into account properly the coupling between the electronic state of the solute and the structure of the solvent). Additional approximations related are needed in order that the FDET-based non-uniform model remains computationally attractive. These aspects were discussed in detail in ref. [21]. Table 1 collects the solvatochromic shifts in absorption and emission calculated with most realistic approach where effect of solvent on geometry and the charge distribution of the solvent are considered.

The FDET embedding potential is not the same for ground- and excited states of the solvated chromophore. Since it is a functional of the charge densities (see Eqn. (3)), it changes if either of the two densities $\left(\rho_{A}\right.$ or $\left.\rho_{B}\right)$ changes. This applies not only

Table 1. Solvatochromic shifts ${ }^{a}$ (in [eV]) of $\pi \rightarrow \pi^{*}$ absorption and emission bands of coumarin 153. Details of the FDET based non-uniform continuum model calculations can be found in ref. [21].

\begin{tabular}{|c|c|c|c|c|c|}
\hline \multirow[t]{2}{*}{ Solvent } & \multirow[t]{2}{*}{$\varepsilon$} & \multicolumn{2}{|l|}{ Absorption } & \multicolumn{2}{|l|}{ Emission } \\
\hline & & $\begin{array}{l}\text { Non-uniform } \\
\text { continuum model }^{\text {b }}\end{array}$ & $\begin{array}{l}\text { Experi- } \\
\text { ment }^{\mathrm{a}}\end{array}$ & $\begin{array}{l}\text { Non-uniform } \\
\text { continuum model }^{\text {b }}\end{array}$ & $\begin{array}{l}\text { Experi- } \\
\text { ment }^{\mathrm{a}}\end{array}$ \\
\hline Water & 78 & -0.29 & $-0.28^{c}$ & -0.49 & $-0.46^{\mathrm{c}}$ \\
\hline Acetonitrile & 38 & -0.19 & $-0.20^{c}$ & -0.36 & $-0.38^{c}$ \\
\hline Ethanol & 25 & -0.23 & $-0.24^{\mathrm{d}}$ & -0.40 & $-0.45^{\mathrm{d}}$ \\
\hline Acetone & 21 & -0.21 & $-0.22^{\mathrm{d}}$ & -0.40 & $-0.38^{\mathrm{d}}$ \\
\hline Diethyl ether & 4 & -0.14 & $-0.1^{\mathrm{d}}$ & -0.32 & $-0.18^{\mathrm{d}}$ \\
\hline
\end{tabular}

aExperimental shifts are given with respect to maximum of the band measured in the least polar

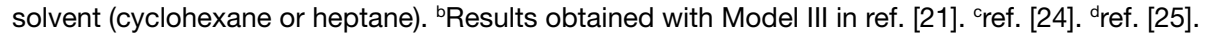



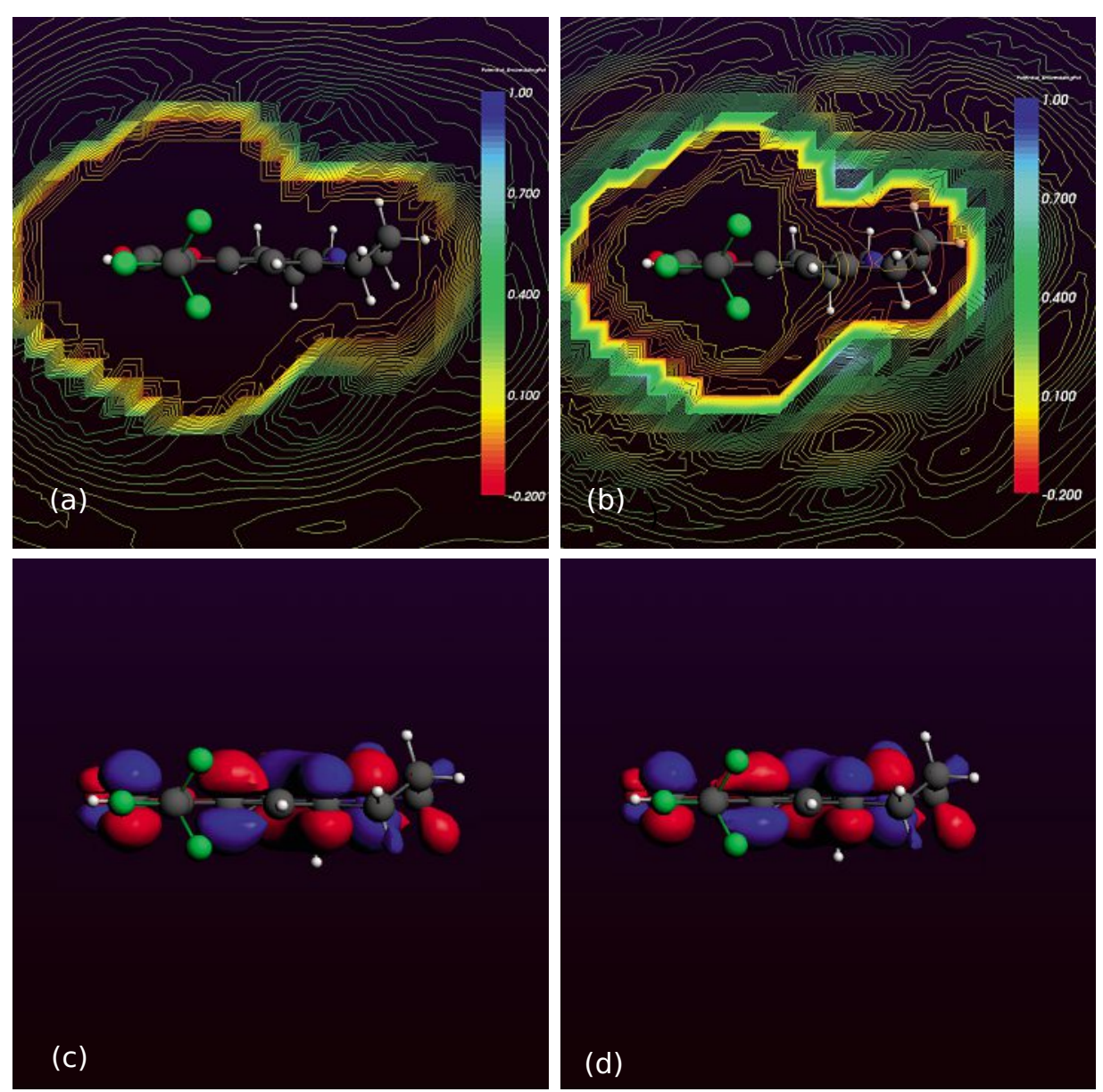

Fig. 1. Top panel: embedding potential (in atomic units) for solvated coumarin 153 in: a) cyclohexane and b) water. Bottom panel: Highest Occupied Embedded Molecular Orbital for coumarin 153 in: c) cyclohexane and d) water. See ref. [7] for details of the calculation.

for the case where $\rho_{B}$ is a density of some polyatomic system in Born-Oppenheimer approximation but also if $\left\langle\rho_{\mathrm{B}}\right\rangle$ is used in FDET. The issue of numerical importance of such dependencies in case of a fixed geometry of the whole system was first analyzed in detail in ref. [26]. In the case of the model considered here, the change of $\left\langle\rho_{B}\right\rangle$ reflecting the change in the average structure of the solvent upon excitation contributes also to the state specificity of the embedding potential. The embedding potentials for ground- and excited state of coumarin 153 in five solvents of different polarity are plotted in Fig. 2. Two representative orbitals involved in the considered excitation for isolated coumarin 153: the highest occupied orbital (HOMO) in the ground-state geometry and the lowest unoccupied molecular orbital (LUMO) in the excited state geometry are also shown. Both orbitals have $\pi$ character. HOMO is spread over the whole chromophore while LUMO features big lobes in one edge of the molecule (see Fig. 2) that includes the carbonyl group, and no significant contribution from the atoms in the opposite edge. For hydrogen bonding solvents (water and ethanol) the embedding potential changes color noticeably from right side of the mol- ecule to the left side in Fig. 2. It is highly attractive (high density of yellow lines) in the vicinity of the carbonyl group in both the ground- and excited state. As a result orbital energies are lowered in the ground and excited states. In this case the zero line passes through the center and left side of the plot (Figure 2 (a) and (d)). In the excited state of the molecule the HOMO and LUMO energies are lowered by $0.3 \mathrm{eV}$ and $0.9 \mathrm{eV}$ respectively when the chromophore is in water, thus the HOMO-LUMO gap is lowered, which is reflected in a higher negative solvatochromic shift in emission compared to absorption. On careful inspection of Fig. 2 one can see that the embedding potential is different inside the cavity for ground- and excited states of $\mathrm{C} 153$. The isopotential lines inside the cavity lie closer to each other in the case of the excited state of the molecule than in the ground state. The orbital-specific stabilization or destabilization effect on frontier orbitals can be, therefore, expected. For the least structured solvent (diethyl ether), the embedding potential is relatively smooth. It varies more in the molecular interior than in the case of cyclohexane (compare Figs 1 and 2) but less than in other solvents. In the ground state the HOMO and LUMO energy is shifted up by $0.04 \mathrm{eV}$ and $0.1 \mathrm{eV}$ respectively, in contrast in the excited state these are lowered by $0.1 \mathrm{eV}$ and $0.2 \mathrm{eV}$. As a result, the discriminating effect of the embedding potential on different orbitals, which lays at the origin of the solvatochromic shifts, is smaller than in more polar solvents but still larger than in cyclohexane. This is reflected in the magnitude of the solvatochromic shifts collected in Table 1.

The maps of average charge density (see Fig. 3) show a gradual appearance of features, which reflect specific solventsolute interactions (hydrogen-bonding to the carbonyl group of the solute in the case of water and methanol). In diethyl ether, the average net charge density is more uniform. Compared to the water and ethanol, local maxima and minima in the maps of the average charge density are smeared out and reflect just the existence of solvatation shells. In the case of water, the positively charged region close to the carbonyl group reflects the hydrogen bonding in that region. Three positively charged lobes near the carbonyl oxygen represent the three possible arrangements of hydrogen bonds. Further away from the carbonyl, the negatively charged domain is due to the oxygen atoms of water molecules involved in the $\mathrm{C}=\mathrm{O} \ldots \mathrm{H}-\mathrm{O}$ bond. At first glance, the ground-state and excited-state maps do not differ noticeably. This indicates that the redistribution of electron density of the chromophore following electronic excitation only negligibly affects the average structure of the solvent. In the case of water, a small effect can be noted. Excitation of the solute results in a small accumulation of the positive charge near the carbonyl. For the maps of net charge densities for other solvents see refs. [7, 21].

\section{Conclusions}

Previous work showed that the FDETbased non-uniform continuum model of the solvent is an attractive computational approach to evaluate solvatochromic shifts in UV/Vis absorption. ${ }^{[6,7]}$ The results for emission, which involves additional approximations, are encouraging. ${ }^{[21]}$ As in commonly used continuum uniform dielectric models of the solvent, only two calculations of the excitation energies for a given geometry of the chromophore are required to obtain the numerical value of the solvatochromic shift. Such methods do not provide, therefore, any information about inhomogeneous broadening. The statistical nature of the system is taken into account implicitly. Compared to the continuum uniform dielectric models, the use of the average electron density of the solvent $\left\langle\rho_{B}\right\rangle$, makes it possible to take 
Ground state
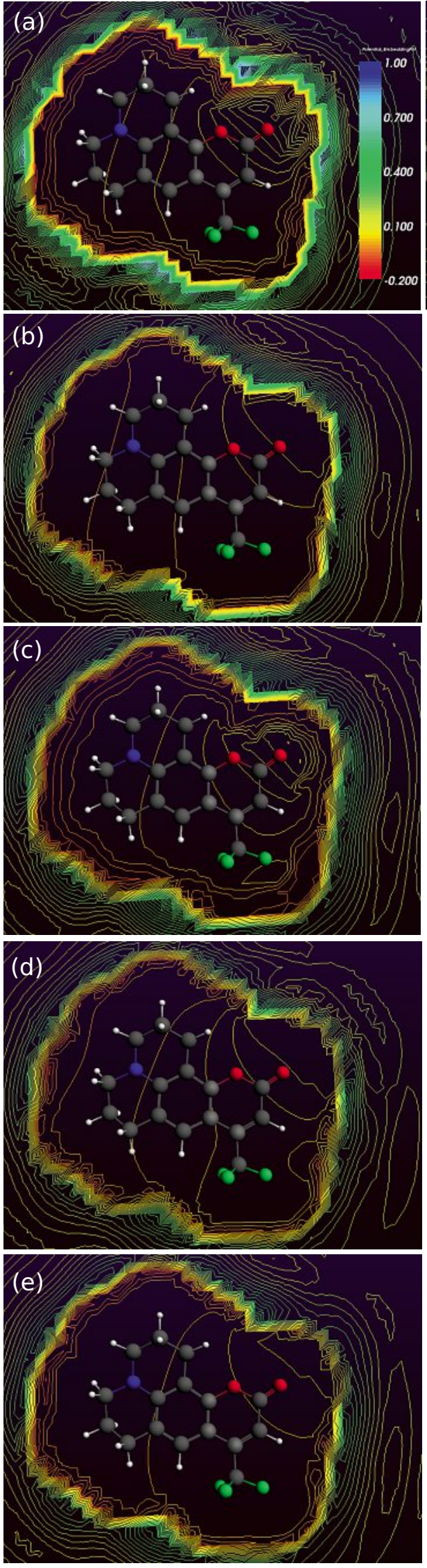

HOMO

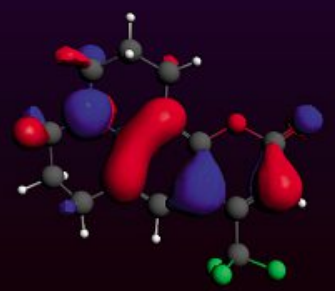

Excited state
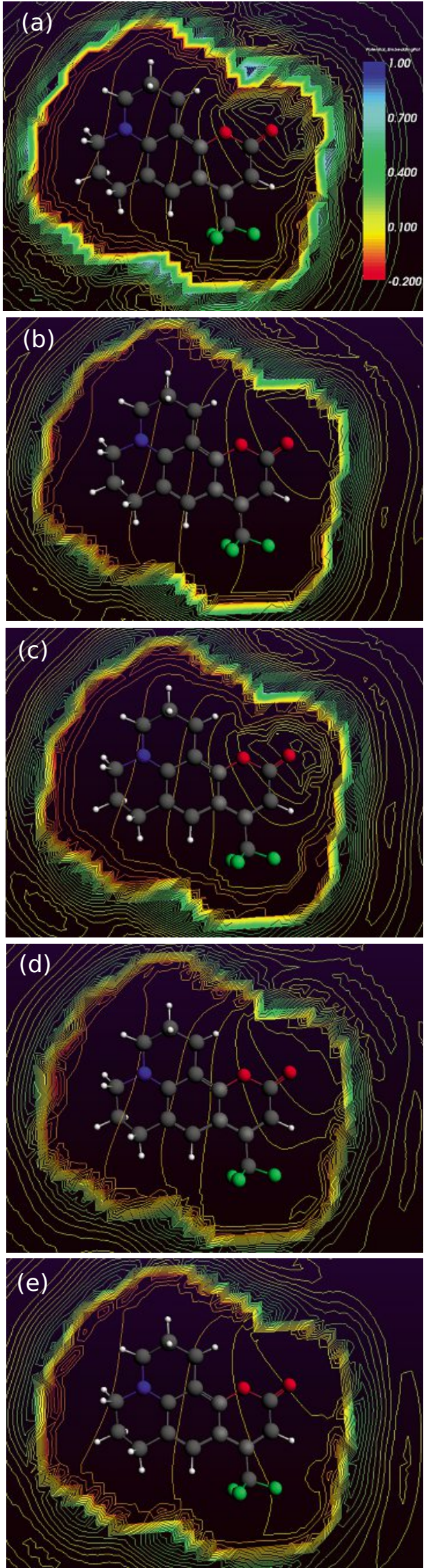

LUMO

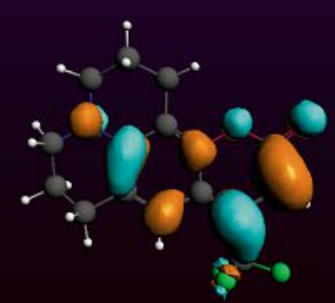

Fig. 2. Embedding potential (in atomic units) for ground- and excited state of coumarin 153 in a) water, b) acetonitrile c) ethanol, d) acetone, and e) diethyl ether. The highest occupied embedded molecular orbital for the ground state and the lowest unoccupied embedded molecular orbital for excited state are shown at the bottom of the figure. The same scale of color bar is used for plotting potentials in all solvents. See ref. [21] for details of the calculation.

into account specific solvent-solute interactions. The present work focused on interpretational value of quantities used in this model: the average charge densities and embedding potential. The maps of these quantities were shown to provide an easy to use tool for qualitative analysis of the origin of solvatochromic shifts. The applications of the principal approximation in the considered, i.e. the use of averaged density $\left\langle\rho_{\mathrm{B}}\right\rangle$ in FDET, are not limited to solvents. The model is also suited for other cases featuring a relatively rigid chromophore surrounded by a structurally flexible environment. This includes such interesting systems as chromophores embedded in: i) solvents at solid-liquid interfaces, ii) solvents confined in porous solids, and iii) structurally flexible polymers (chromophores surrounded by flexible side chains in proteins), for instance. We plan several applications for such systems. The applications overviewed in the present work provide the necessary benchmark reference data for the planned applications.

\section{Acknowledgements}

This research was supported by grants from Swiss National Science Foundation (Project 200021_152779).

Received: June 18, 2014

[1] a) J. Zeng, N. S. Hush, J. R. Reimers, J. Chem. Phys. 1993, 99, 1508; J. Gao, M. Freindorf, J. Phys. Chem. A 1997, 101, 3182; b) U. F. Röhrig, I. Frank, J. Hutter, A. Laio, J. VandeVondele, U. Rothlisberger, Chem. Phys. Chem. 2003, 4, 1177; c) J. Kongsted, A. Osted, T. B. Pedersen, K. V. Mikkelsen, J. Phys. Chem. A 2004, 108 , 8624; d) C. Zazza, A. Amadei, N. Sanna, A. Grandi, G. Chillemi, A. Di Nola, M. D’Abramo, M. Aschi, Phys. Chem. Chem. Phys. 2006, 8, 1385; e) S. Yoo, F. Zahariev, S. Sok, M. S. Gordon, J. Chem. Phys. 2008, 129, 144112; f) J. M. Olsen, K. Aidas, K. V. Mikkelsen, J. Kongsted, J. Chem. Theory Comput. 2009, 6, 249.

[2] J. Neugebauer, C. R. Jacob, T. A. Wesolowski, E.-J. Baerends, J. Phys. Chem. A 2005, 109, 7805.

[3] a) J. Neugebauer, M. J. Louwerse, E.-J. Baerends, T. A. Wesolowski, J. Chem. Phys. 2005, 122, 09411; b) X. Zhou, T. A. Wesolowski, G. Tabacchi, E. Fois, G. Calzaferri, A. Devaux, Phys. Chem. Chem. Phys. 2013, 15, 159.

[4] B. Mennucci, J. Tomasi, J. Chem. Phys. 1997, $106,5151$.

[5] A. Klamt, G. Shuurmann, J. Chem. Soc. Trans. 1993, 2, 799 . 


\section{Ground state}
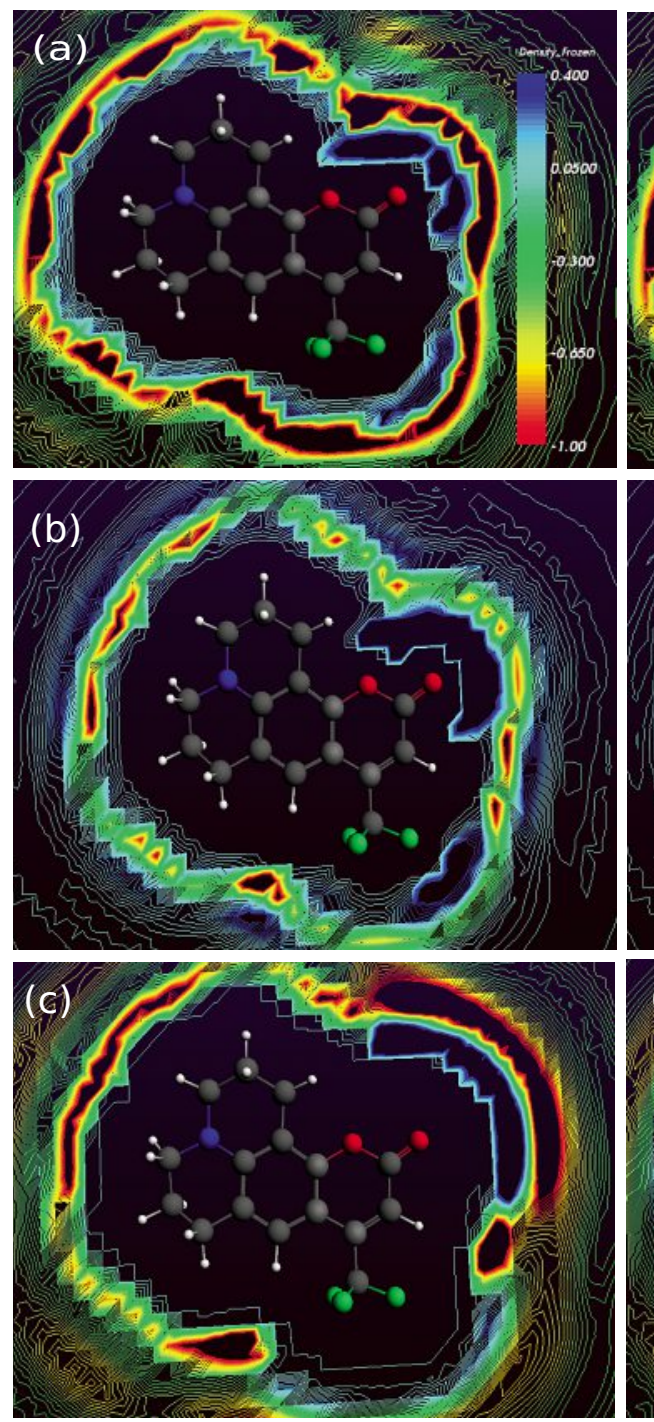
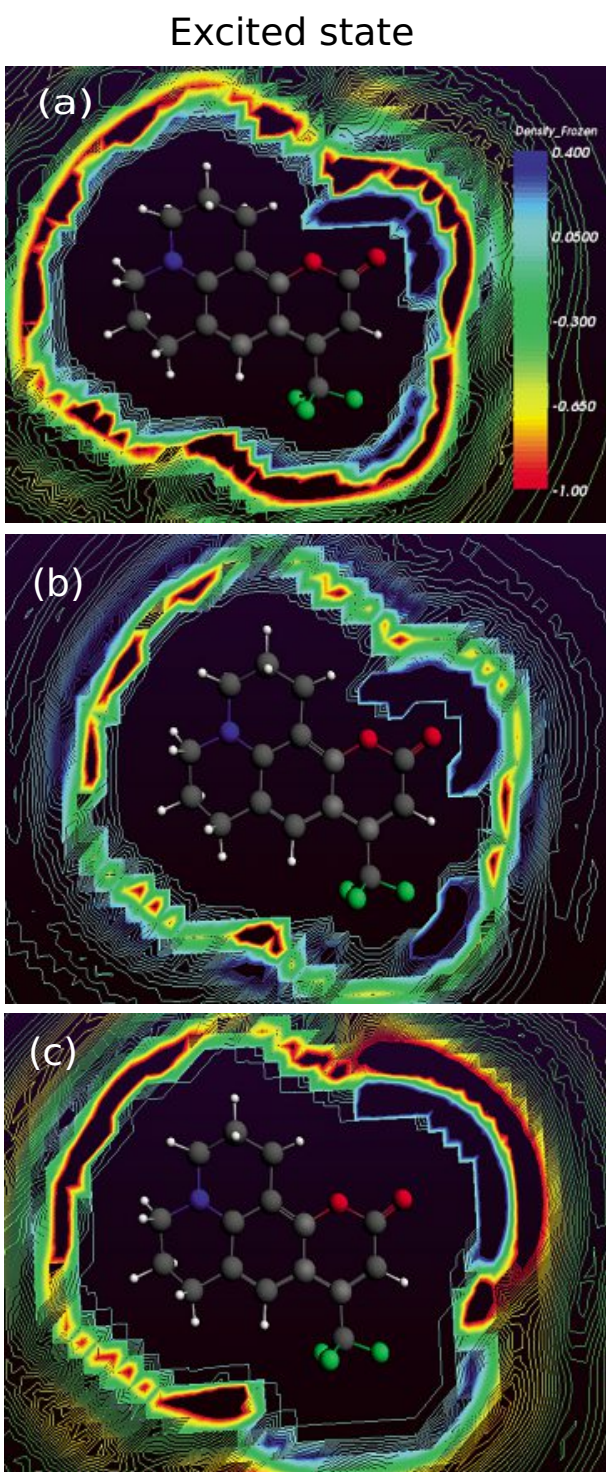

Fig. 3. Density of the net average charge for a) water $(\varepsilon=78)$, b) ethanol $(\varepsilon=25)$, and c) diethyl ether $(\varepsilon=4)$ for ground- and excited state of coumarin 153 (in atomic units). Due to rapid variation of $\left\langle\rho_{\mathrm{B}}\right\rangle$ the plots show the net average charge which includes also the average nuclear charge (quickly varying positive function).

[6] J. W. Kaminski, S. Gusarov, T. A. Wesolowski, A. Kovalenko, J. Phys. Chem. A, 2010, 114, 6082.

[7] X. Zhou, J. W. Kaminski, T. A. Wesolowski, Phys. Chem. Chem. Phys. 2011, 13, 10565.

[8] T.A. Wesolowski, A. Warshel, J. Phys. Chem. 1993, 97, 8050

[9] T.A. Wesolowski, Phys. Rev. A 2008, 77, 012504.

[10] P. Hohenberg, W. Kohn, Phys. Rev. 1964, 136, B864.

[11] W. Kohn, L. J. Sham, Phys. Rev. 1965, 140, A1133.

[12] M. Levy, Proc. Natl. Acad. Sci. 1979, 76, 6062.

[13] Y. G. Khait, M. R. Hofmann, J. Chem. Phys. 2010, 133, 044107.

[14] T.A. Wesolowski, J. Chem. Phys. 2014, 140, 18A530.

[15] T.A. Wesolowski, J. Am. Chem. Soc. 2004, 126, 11444.

[16] a) T. Kluner, N. Govind, Y. A. Wang, E. A. Carter, Phys. Rev. Lett. 2001, 86, 5954; b) T. Kluner, N. Govind, Y.A. Wang, E.A. Carter, J. Chem. Phys. 2002, 116, 42.

[17] M. E. Casida, 'Time-dependent densityfunctional response theory for molecules', in 'Recent Advances in Density Functional Methods', World Scientific, Singapore, 1995, vol. 1, pp. 155-192.

[18] M.E. Casida, T.A. Wesolowski, Int. J. Quantum Chem. 2004, 96, 577.

[19] J. Neugebauer, Phys. Rep. 2010, 489, 1.

[20] M. Hidalgo, R. Rivelino, S. Canuto, J. Chem. Theory Comput. 2013, 10, 1554.

[21] S. V. Shedge, T. A. Wesolowski, ChemPhysChem, 2014, in press.

[22] A. Kovalenko, 'Three-dimensional RISM theory for molecular liquids and solid-liquid interfaces', in 'Understanding Chemical Reactivity', Kluwer Acedemic Publishers, Dordrecht, The Netherlands, 2003, vol. 24, pp. 169-275; A. Kovalenko, F. Hirata, J. Chem. Phys. 1999, 110, 10095.

[23] GUI 2013, SCM, Amsterdam, The Netherlands, http://www.scm.com.

[24] P. Hazra, D. Chakrabarty, N. Sarkar, Chem. Phys. Lett. 2003, 371, 553.

[25] M. L. Horng, J. A. Gradecki, A Papazyan, M. Maroncelli, J. Phys. Chem. 1995, 99, 17311.

[26] C. Daday, C. König, O. Valsson, J. Neugebauer, C. Filippi, J. Chem. Theory Computation 2013, 9, 2355. 